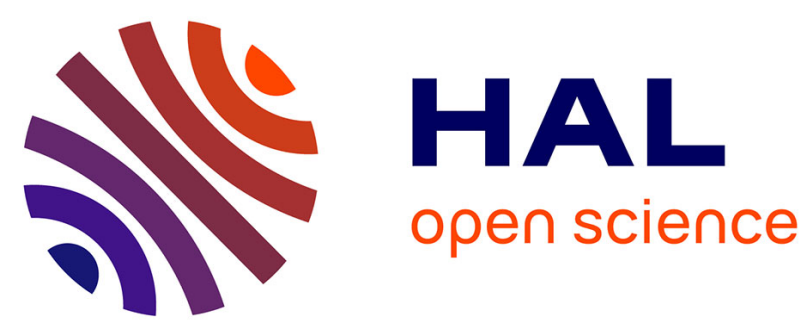

\title{
Syntactic texture and perception for a new generic visual anomalies classification
}

Simon-Frédéric Desage, Gilles Pitard, Maurice Pillet, Hugues Favrelière, Jean-Luc Maire, Fabrice Frelin, Serge Samper, Gaëtan Le Goïc

\section{To cite this version:}

Simon-Frédéric Desage, Gilles Pitard, Maurice Pillet, Hugues Favrelière, Jean-Luc Maire, et al.. Syntactic texture and perception for a new generic visual anomalies classification. Quality Control by Artificial Vision (QVAV), Jun 2015, Le Creusot, France. 10.1117/12.2182819 . hal-02136841

\section{HAL Id: hal-02136841 \\ https://hal.science/hal-02136841}

Submitted on 22 May 2019

HAL is a multi-disciplinary open access archive for the deposit and dissemination of scientific research documents, whether they are published or not. The documents may come from teaching and research institutions in France or abroad, or from public or private research centers.
L'archive ouverte pluridisciplinaire HAL, est destinée au dépôt et à la diffusion de documents scientifiques de niveau recherche, publiés ou non, émanant des établissements d'enseignement et de recherche français ou étrangers, des laboratoires publics ou privés. 


\title{
Syntactic texture and perception for a new generic visual anomalies classification
}

\author{
Simon-Frédéric Désage ${ }^{a}$, Gilles Pitard ${ }^{a}$, Maurice Pillet $^{a}$, Hugues Favrelière ${ }^{a}$, Jean-Luc Maire $^{a}$, \\ Fabrice Frelin $^{a}$, Serge Samper $^{a, b}$ and Gaëtan Le Goïc ${ }^{a, c}$ \\ ${ }^{a}$ SYMME, Laboratoire des Systèmes et Matériaux pour la Mécatronique, Université de Savoie \\ Mont-Blanc, F-74000 Annecy, France; \\ ${ }^{b}$ LARMAUR - ERL CNRS 6274, Laboratoire de Recherche en Mécanique Appliquée de \\ l'Université de Rennes 1, F-35000 Rennes, France; \\ ${ }^{c}$ LE2I, Laboratoire d'Electronique, Informatique et Image, UMC CNRS 6306, Université de \\ Bourgogne, F-89000 Auxerre, France
}

\begin{abstract}
The research purpose is to improve aesthetic anomalies detection and evaluation based on what is perceived by human eye and on the 2006 CIE report. ${ }^{1}$ It is therefore important to define parameters able to discriminate surfaces, in accordance with the perception of human eye. Our starting point in assessing aesthetic anomalies is geometric description such as defined by ISO standard, ${ }^{2}$ i.e. traduce anomalies description with perception words about texture divergence impact. However, human controllers observe (detect) the aesthetic anomaly by its visual effect and interpreter for its geometric description. The research question is how define generic parameters for discriminating aesthetic anomalies, from enhanced information of visual texture such as recent surface visual rendering approach. We propose to use an approach from visual texture processing that quantify spatial variations of pixel for translating changes in color, material and relief. From a set of images from different angles of light which gives us access to the surface appearance, we propose an approach from visual effect to geometrical specifications as the current standards have identified the aesthetic anomalies.
\end{abstract}

Keywords: visual inspection, classification, Surface imperfection, geometrical description, visual effect, visual texture

\section{INTRODUCTION}

\subsection{Motivation}

Nowadays, perceived quality of visual appearance is [still] an industrial matter. The main difficulty is to link objective measurements and subjective human aesthetic indicators. The CIE (International Commission on Illumination) provided a technical report CIE 175:2006 "A framework for the measurement of visual appearance". ${ }^{1}$ This report defines four headings under which possible measures might be made: color, gloss, translucency and texture. However, when a customer, and thus imitating his behavior, a human controller, inspect the visual appearance of a product, they can not differentiate the four parties. Considering color and translucency as known and controlled by the industry, we have chosen to work on the gloss and texture. The goal is to identify visual - aesthetic anomalies and traduce some geometric considerations from ISO standard 8785:1998 ${ }^{2}$ to visual effects considerations. A known difficulty is the interdependence of these measurements because translucency can influence color, which may influence gloss, and texture is probably a combination of all three. ${ }^{4}$ One work point is to differentiate [human] detection and perception, knowing that it is necessary to combine both steps to enable better decision about quality inspection. Indeed, quality inspection is divided into three steps: Exploration, Evaluation and Decision ${ }^{5},{ }^{6}$ The main difference between a classic vision system and a human controller is

Further author information: (Send correspondence to Simon-Frédéric Désage)

E-mail: simon-frederic.desage@univ-savoie.fr

E-mail: \{gilles.pitard, maurice.pillet, hugues.favreliere, jean-luc.maire, fabrice.frelin\}@univ-savoie.fr

E-mail: serge.samper@univ-rennes1.fr and gaetan.le-goic@u-bourgogne.fr 
perception notions in Evaluation step. There is some anomaly elements strongly detected but weakly perceived, and conversely, some anomaly elements weakly detected and strongly perceived. We have chosen to present in this paper a proposal for improvement of the syntactic description of surface imperfections to prepare the evaluation. This description must be more perceptual and must take into account the visual impact of anomalies. Our starting technical point is the exploitation of the most advanced visual surface representation and image processing approach of texture classification. ${ }^{7}$ Our starting theorical determination is the study and the imitation of human behavior in the surface quality inspection (Figure 1).

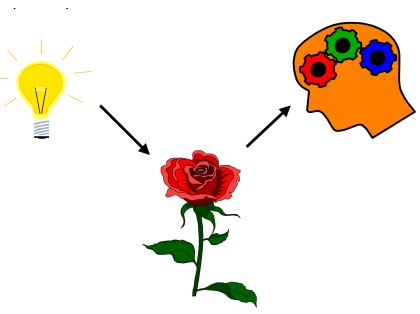

Figure 1: The vision scheme from $\mathrm{CIE}^{1}$

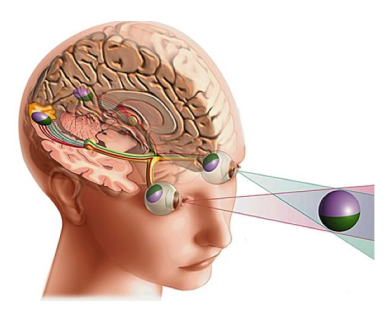

Figure 2: The human visual system for surface inspection. Credit: Michel Saemann for Larousse

\subsection{Methodology}

There are already many research and industrial solutions exploiting anomaly appearance descriptions, but these are case by case solutions with suitable devices (limited lighting and viewing conditions). We find examples in various fields of applications, such as for controlling the road conditions ${ }^{8}$ or the appearance of the fruits, ${ }^{9}$ or to control the textile manufacturing quality. ${ }^{10}$

This work proposes a generic [perception-based] visual anomalies classification, like a bridge between geometrical specifications from dimensional metrology and surface appearance specifications of aesthetic anomalies. The research purpose is double. Firstly, following quality inspection formalization based on visual human behavior and providing complementary indicators of detection and perception, there is a hope to provide a better understanding of visual human mechanisms. Secondly, there is a desire to reduce the surface anomaly classification to the perceptual classification and to provide a robust imitation of controller for detection and evaluation of aesthetic anomalies. The syntactic appearance classification is based on texture attributes, such as the tone (light, dark), the roughness (rough, smooth, rippled), the light aspect (matte, speckled), the finesse (fine, coarse) and the regularity (regular or irregular). By example, scratch is viewed like a white line, i.e. a fine anomaly with a regular, smooth, light tone and speckled aspect. ${ }^{11}$

In short, there are two steps to construct a new visual anomalies classification. Firstly, we need to provide an exhaustive restricted classification, based on geometrical considerations of the standards for surface imperfections. Secondly, we need to describe all anomalies by their visual effects. We propose then some considerations from new visual texture processing to identify the presence or not of an anomaly.

\section{CLASSIFICATION OF AESTHETIC ANOMALIES}

Then the work is composed of two parts, similar to the human visual system. The first part is to describe the visual impression (or stimulus) of appearance anomalies with a pattern recognition mechanism. Indeed, the first part of the human visual system is associated with the eye and its ability to detect and pattern recognition learned by individual culture. This work is based on the standards of geometrical product specifications ${ }^{2}$ as the human capacity for interpretation allows visual recognition from the causal description of an anomaly. These norms are used to define the set of search anomalies and associated criteria. The second part is to put into perspective the shape or patterns recognized with an ambient context to perceptual evaluation interdependent with the surrounding visual texture (object context). 


\subsection{Geometrical classification and standard}

A finding of our previous work is that the vocabulary designating surface imperfections among industrial companies, whether or not in the same domain is rarely the same. This difficulty leads to potential misunderstandings because how do we know if we talk about the same thing. We have chosen to focus the first part of work from the ISO $8785-1998^{2}$ dealing with surface imperfections. This standard provides anomalies terms, definitions and parameters from causal descriptions. We establish an initial classification of visual anomalies following their morphological and appearance features. Our starting point is the 31 cases outlined in the standard ${ }^{2}$ summa- $^{-}$ rized in a table (Figure 3) to have a global view of all surface imperfections, considering that the standard $^{2}$ is comprehensive.

\begin{tabular}{|c|c|c|c|c|c|c|}
\hline Number & Name & Planar shape & Relief shape & Density & Relative size scale & Appearance contrast \\
\hline 1 & Groove & Linear & In & One & Micro-Medium & Medium - Light \\
\hline 2 & Scratch & Linear & In & One & Micro-Medium & Medium - Light \\
\hline 3 & Crack & Linear & In & One & Micro-Medium & Dark-Medium \\
\hline 4 & Pore & Circular & In & One - Many & Micro & Dark-Medium \\
\hline 5 & Blowhole & Circular & In & One - Many & Micro-Medium & Dark-Medium \\
\hline 6 & Shrinkage hole & Circular & In & One & Medium & Dark - Medium \\
\hline 7 & Fissure / Chink / Crevice & Linear & In & One - Many & Micro-Medium & Medium - Light \\
\hline 8 & Wane & Linear & In & One & Medium & Medium \\
\hline 9 & (concave) Buckle & Curved & In & One & Medium & Dark-Medium \\
\hline 10 & Dent & Circular & In & One & Medium & Dark-Medium \\
\hline 11 & Wart & Linear & Out & One - Many & Micro-Medium & Medium - Light \\
\hline 12 & Blister & Circular & Out & One - Many & Micro-Medium & Medium - Light \\
\hline 13 & (convex) Buckle & Curved & Out & One & Medium & Medium - Light \\
\hline 14 & Scale & Linear & Out & One & Micro-Medium & Dark - Medium \\
\hline 15 & Inclusion & Circular & Medium - Out & One - Many & Micro-Medium & All \\
\hline 16 & Burr & Linear & Out & One & Medium & Medium - Light \\
\hline 17 & Flash & Linear & Out & One & Medium & Medium - Light \\
\hline 18 & Deposits & All & Out & One - Many & Micro-Medium & All \\
\hline 19 & Crater & Circular & All & One - Many & Micro-Medium & All \\
\hline 20 & Lap & Curved & Out & One & Micro-Medium & Medium - Light \\
\hline 21 & Scoring & All & All & One & Micro - Medium & All \\
\hline 22 & Chip rest & Linear & Out & One - Many & Micro-Medium & Medium - Light \\
\hline 23 & Skidding & Circular & Medium & One - Many & Micro-Medium & All \\
\hline 24 & Erosion & Linear & In - Medium & One - Many & Micro-Medium & All \\
\hline 25 & Corrosion & All & Medium & One - Many & Micro-Medium & All \\
\hline 26 & Pitting & Circular & Medium & One - Many & Micro & Medium - Light \\
\hline 27 & Crazing & Linear & Medium & One - Many & Micro-Medium & All \\
\hline 28 & Spot / Patch & All & Medium & One - Many & Micro-Medium & All \\
\hline 29 & Discoloration & All & Medium & One - Many & Micro-Medium & All \\
\hline 30 & Streak & Linear & In - Medium & One - Many & Medium & Dark - Medium \\
\hline 31 & Cleavage / Flaking & All & Medium & One - Many & Micro-Medium & All \\
\hline
\end{tabular}

Figure 3: Table of ISO 8785 Anomalies Classification from their causal descriptions

We propose to use the following five simple scales to classify these imperfections. These scales are illustrated by the figure 4 , respectively.

1. First rule : planar shape - From linear to circular. This rule is calculated based on the circularity of the bounding ellipse of the anomaly. Indeed, from this ellipse, we can extract the eccentricity, which is the ratio of the two axes of the ellipse. If it tends to 1 then the anomaly tends to look like a circle, while conversely, if the eccentricity tends to 0 , then the anomaly will be linear.

2. Second rule : relief shape - From inwardly to outwardly. This rule measures the average topographic impact of the anomaly, ie if it has more removed or added to the material from the model plan. It allows to differentiate mainly hollow compared to the bumps.

3. Third rule : density - From one to infinity (a number - many). This rule measures the amount of small anomalies that constitutes a group such as we consider only a single visual anomaly.

4. Fourth rule : relative size scale - From microscopic to object scale. We study small parts size of ten centimeters maximum. Our size scale varies from mesoscopic to macroscopic, ie from the smallest visible by a human eye to a size consistent with the size of the observed part. The parts considered to be of good quality, we do not observe the anomalies consistent with the size of the part, but only at lower scales and mostly microscopic. 
5. Fifth rule : appearance contrast scale - From dark to light via contrast. It is sometimes difficult to judge whether a part of the room is darker or lighter than its surroundings, considering the changes in his appearance for different lighting configurations / surface / viewpoint. In our approach, we consider appearance characteristic of the anomaly when is highlighted with respect to its neighborhood. For example, a scratch can vary from light to dark as the relative positioning illumination / view, but it is primarily highlighted when it shines compared to its neighborhood. In this case, we consider its appearance as light, in contrast to its neighbors.

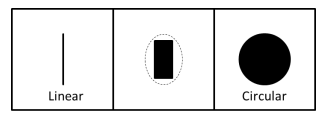

(a)

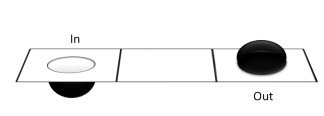

(b)

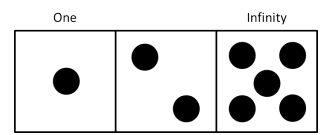

(c)

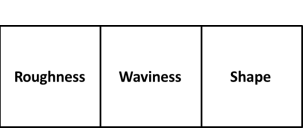

(d)

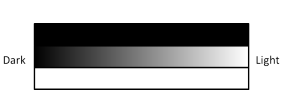

(e)

Figure 4: The five appearance rules to describe surfaces imperfections from ISO 8785 - 1999 (a) The scale of circularity criterion for planar shape, i.e. the eccentricity of ellipse bounding (b) The scale of relief criterion, i.e. the mean relief of anomaly (c) The scale of density, i.e. the number of labels (d) the relative scale of anomaly compared to object (e) the contrast scale compared to the neighborhood of anomaly

We can provide some clarifications to the scale on the relative size of the anomaly, from considerations from the dimensional metrology. ${ }^{12}$ Indeed, as shown in the figure 5, it is usual to consider four orders of composition as a macroscopic object, corresponding to the shape, waviness, roughness and microroughness. In our approach, we merge the two categories of roughness, especially because it can be difficult to discern both visually for a human without additional tools.

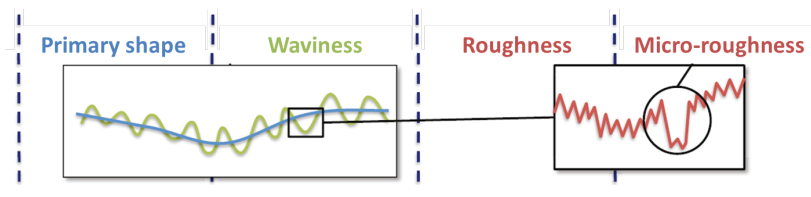

Figure 5: The 4 orders scale of surface imperfections considered in dimensional metrology ${ }^{12}$

To improve the visualization of anomaly characterization, we propose to use a Kiviat diagram or star diagram (figure 6) to describe the set of solutions for each anomaly. To simplify the classification, we use only three levels with both previous named extremums. For example, we applied this representation for the groove case (figure 7) and the pattern show the solutions set for groove-type anomalies. The advantage of this representation is that it allows you to associate a pattern to an anomaly, which is easier perceptually than a sentence to distinguish and compare, because it can be independent of the language used.

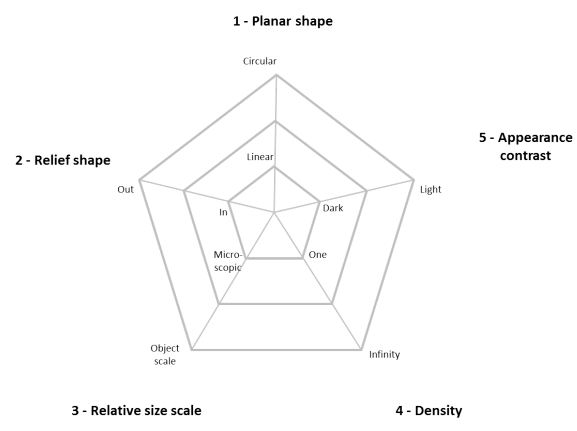

Figure 6: Primary Kiviat evaluation diagram for anomaly

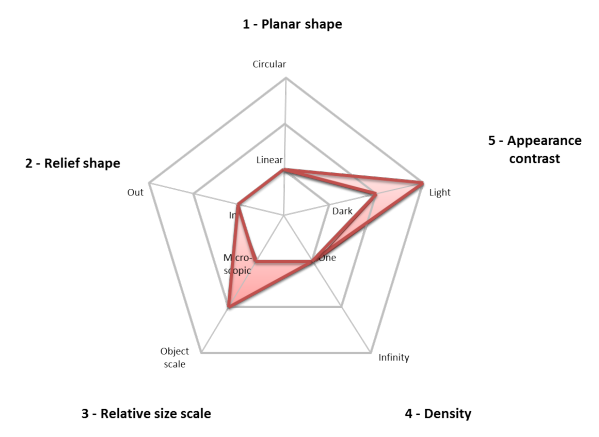

Figure 7: Evaluation for groove-like anomaly following the five previous levels. 
If we consider at least three levels by previous classification rules, there are at least $3^{5}$ cases, namely 243 cases. The standard allows to summarize all the case with just over $10 \%$ of cases (13\%). Nevertheless we still want to reduce the classification of aesthetic anomalies relative to the geometric perception, to establish a generic and comprehensive automatic detection.

\subsection{Restricted classification by geometrical perception}

Previous work ${ }^{13,14}$ has shown the possibility of reducing and optimizing only 4 types (perceptual descriptions) of anomalies, and virtually no loss in detection of surface appearance anomalies. These four categories are:

1. Mark: Any trace left on the surface of the part representing a lack of timely matter.

2. Deformation: Any change in shape, altering the initial shape.

3. Heterogeneity: Any change in spot color on the surface, shadow or cloud.

4. Pollution: A foreign body.

There is also a higher order than these four groups to distinguish between progessive or non anomalies. In our approach, we consider only those that are not. However, if it does not affect the anomaly detection method, it has an increased importance in the perception of scalable anomalies. Indeed, scalable anomalies are at risk of growing degradation medium or long terms, while non-scalable anomalies have a direct impact on the appearance or product specifications.

\begin{tabular}{|c|c|c|c|c|c|}
\hline Number & Name & Mark & Deviation & Heterogeneity & Pollution \\
\hline 1 & Groove & Yes & No & No & No \\
\hline 2 & Scratch & Yes & No & No & No \\
\hline 3 & Crack & Yes & No & No & No \\
\hline 4 & Pore & Yes & No & No & No \\
\hline 5 & Blowhole & Yes & No & No & No \\
\hline 6 & Shrinkage hole & Yes & No & No & No \\
\hline 7 & Fissure/Chink/Crevice & Yes & No & No & No \\
\hline 8 & Wane & Yes & No & No & No \\
\hline 9 & (concave) Buckle & Yes & No & No & No \\
\hline 10 & Dent & Yes & No & No & No \\
\hline 11 & Wart & No & Yes & No & No \\
\hline 12 & Blister & No & Yes & No & No \\
\hline 13 & (convex) Buckle & No & Yes & No & No \\
\hline 14 & Scale & No & Yes & No & No \\
\hline 15 & Inclusion & No & Possible & Possible & Possible \\
\hline 16 & Burr & No & Yes & No & No \\
\hline 17 & Flash & No & Yes & No & No \\
\hline 18 & Deposits & No & Possible & Possible & Possible \\
\hline 19 & Crater & Possible & Possible & No & No \\
\hline 20 & Lap & No & Yes & No & No \\
\hline 21 & Scoring & Possible & Possible & No & No \\
\hline 22 & Chip rest & No & Yes & No & No \\
\hline 23 & Skidding & No & No & Possible & Possible \\
\hline 24 & Erosion & Possible & No & Possible & No \\
\hline 25 & Corrosion & No & No & Possible & Possible \\
\hline 26 & Pitting & No & No & Possible & Possible \\
\hline 27 & Crazing & No & No & Possible & Possible \\
\hline 28 & Spot/Patch & No & No & Yes & No \\
\hline 29 & Discoloration & No & No & Yes & No \\
\hline 30 & Streak & Possible & No & Possible & Possible \\
\hline 31 & Cleavage / Flaking & No & No & Possible & Possible \\
\hline & & & & & \\
\hline & & & & \\
\hline & & & & & \\
\hline & & & & & \\
\hline & & & &
\end{tabular}

Figure 8: Restricted classification according to 4 groups $^{13}$

The main risk of excessive reduction of descriptors is the loss of information that may result in the product description. The main additional information necessary for a complete classification is the importance of the anomaly by the surface (the area of influence) and density.

The figure 8 shows the surface imperfections from ISO 8785 following the 4 restricted classification. The name "Possible" means that according to observed anomaly, for the same type of anomaly according to ISO 8785 , it can be judged differently according to the restricted classification. The set of solutions of each class of restricted classification, from the first 5 morphological scales, can overlap but is different and is shown in Figures 9 (a), (b), (c) and (d) 


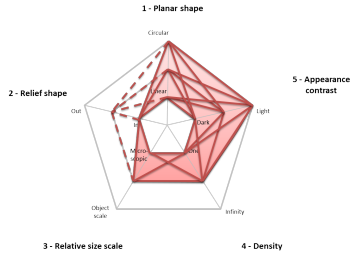

(a)

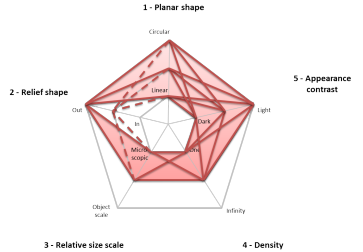

(b)

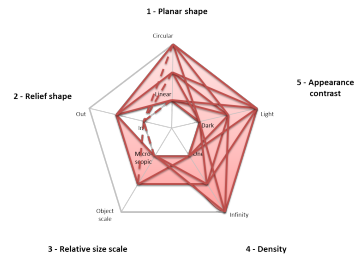

(c)

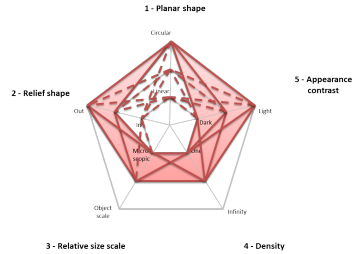

(d)

Figure 9: Correspondence between the 4 groups and initial descriptions. (a) Kiviat diagram for Mark-type anomalies (b) Kiviat diagram for Deviation-type anomalies (c) Kiviat diagram for Heterogeneity-type anomalies (d) Kiviat diagram for Pollution-type anomalies

\section{VISUAL IMPACT AND DETECTION}

However, the aesthetic anomalies are detected by the visual impact of the normal visual behavior deviating from their immediate neighborhood or in relation to a model. It is therefore necessary to identify the influence of the anomalies on the visual appearance of a surface. For this, we consider the Bidimensional Reflectance Distribution Function (BRDF) reflecting the behavior of the visual appearance of surfaces. ${ }^{15}$

\subsection{Classification by visual effects}

For recall, a photography, as image texture, is a visual texture slice and an image is a local result of color, material and relief combination. There is surface texture such as only relief texture, and the surface visual texture is described by a BRDF field.

We consider a new decomposition ${ }^{7}$ of the BRDF associated (Figure 10 (a) and (b)) with all three levels of a surface such as the material, color and relief, reflecting the cause to the expected visual effect. This definition is derived component parameters visual texture, and like a BRDF is a unitary element of a visual texture, so we can decompose it using the same parameters. The shape of BRDF slice is a feature of material, respectively the power/amplitude for the color (Dependent of each wavelength), and the direction for the relief related to the optical law of Snell-Descartes. The amplitude corresponds to the maximum of function and the function direction is the direction of maximum such as the specular direction. In the scratch case, one can expect a relief change from the normal behavior, which will have the effect of influencing the BRDF direction locally.

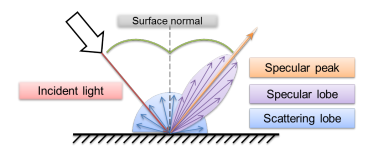

(a)

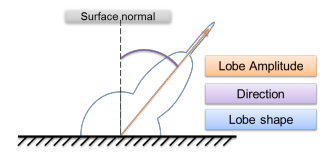

(b)

Figure 10: Decomposition of BRDF (a) Phong model-like BRDF decomposition (b) BRDF decomposition following surface state

There is therefore seven cases of possible variation of visual appearance, considering that if neither the material nor the relief or the color does not change, there is no presence of an anomaly. This state is the reference state. The figure 11 shows all possible cases.

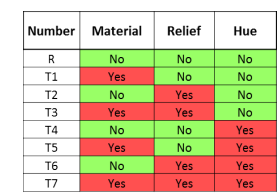

Figure 11: Possible combinations of surfaces states change 
Regardless of what field of possibilities, we propose a classification (Figure 12) of the possible influence of defects on the surface condition. That majority is observed anomalies influence the relief, which correlates with the fact that mainly the techniques used for their detection are the responsibility of the topography. Nevertheless, one can recall the major difficulty of the techniques of topography or dimensional metrology which need to be at the right scale to measure because if sampling of the surface is not good, it can not detect the anomaly. This is the advantage and disadvantage of the main visual detection because the visual effect is "visible" at multiple scales. Indeed, the fact that an aesthetic anomaly is visible at different scales is the fact that it is disturbing. Our classification also provides that certain types of defects can be perceived by different visual effects. The name "Possible" here means "Yes" or "No".

\begin{tabular}{|c|c|c|c|c|c|}
\hline Number & Name & Material & Relief & Hue & Type \\
\hline 1 & Groove & No & Yes & No & T2 \\
\hline 2 & Scratch & No & Yes & No & T2 \\
\hline 3 & Crack & No & Yes & No & T2 \\
\hline 4 & Pore & No & Yes & No & T2 \\
\hline 5 & Blowhole & No & Yes & No & T2 \\
\hline 6 & Shrinkage hole & No & Yes & No & T2 \\
\hline 7 & Fissure / Chink/Crevice & No & Yes & No & T2 \\
\hline 8 & Wane & No & Yes & No & T2 \\
\hline 9 & (concave) Buckle & No & Yes & No & T2 \\
\hline 10 & Dent & No & Yes & No & T2 \\
\hline 11 & Wart & No & Yes & No & T2 \\
\hline 12 & Blister & Possible & Yes & No & T2-T3 \\
\hline 13 & (convex) Buckle & No & Yes & No & T2 \\
\hline 14 & Scale & No & Yes & No & T2 \\
\hline 15 & Inclusion & Yes & Possible & Yes & T5-T7 \\
\hline 16 & Burr & No & Yes & No & T2 \\
\hline 17 & Flash & No & Yes & No & T2 \\
\hline 18 & Deposits & Yes & Possible & Possible & T1-T3-T5-T7 \\
\hline 19 & Crater & No & Yes & No & T2 \\
\hline 20 & Lap & No & Yes & No & T2 \\
\hline 21 & Scoring & Possible & Yes & No & T2-T3 \\
\hline 22 & Chip rest & Possible & Yes & No & T2-T3 \\
\hline 23 & Skidding & Yes & No & Possible & T1-T5 \\
\hline 24 & Erosion & Yes & Possible & No & T1-T3 \\
\hline 25 & Corrosion & Yes & No & Possible & T1-T5 \\
\hline 26 & Pitting & Yes & No & Possible & T1-T5 \\
\hline 27 & Crazing & Yes & No & Possible & T1-T5 \\
\hline 28 & Spot/Patch & Possible & No & Yes & T4-T5 \\
\hline 29 & Discoloration & Possible & No & Yes & T4-T5 \\
\hline 30 & Streak & Yes & Possible & Possible & T1-T3-T5-T7 \\
\hline 31 & Cleavage / Flaking & Yes & No & Possible & T1-T5 \\
\hline
\end{tabular}

Figure 12: Classification by visual impact - Restricted classification according to the change of surfaces states ${ }^{13}$

\subsection{Limits and measurement scale}

A limitation of this approach is related to the scale of measurement. Indeed, depending on the scale, visual effects due to surface condition changes can mingle and merge with the normal behavior of the surface and thus lead to a different interpretation of the first inverse causal link. However, this potential misinterpretation does not lead a priori detection error.

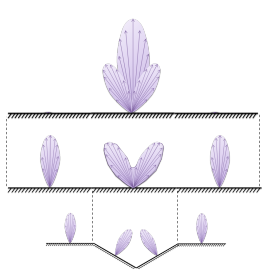

(a)

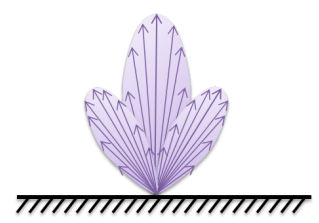

(b)

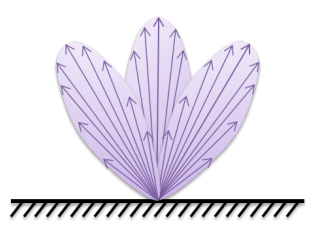

(c)

Figure 13: Visual detection of a scratch illustrated by a slice of BRDF (a) Scale influence on the measured BRDF and scratch detection (b) Scratch with a low contrast (c) Scratch with a high contrast

To illustrate this limit, we have taken the example of a mean scratch (average depth and width) whose visual impact is measured on different scales (Figure $13(\mathrm{a})$ ). Of course, in the real case, we must take into account the optical properties of the material and its color to perform the measurement in good conditions. In our example, the material considered is purely specular, as can be a metal, and there is no chromatic effect. 


\subsection{Measurement of changes and impacts on perceived visual texture}

To measure and identify a deviation of the visual texture, we propose to use a method of analysis texture, similar to the work of Alice Porebski ${ }^{16}$ (Application to flaw identification on decorated glasses printing) which shows the possibility of classification and learning signatures of visual appearance and defects associated from the analysis of a co-occurrence matrix. For this, we rely on the extension of this method to visual texture analysis. ${ }^{7}$

By cons, by the approach of visual texture analysis, it is appropriate to use five new scales related to the texture. ${ }^{1}$ We propose to use the following five simple scales to classify these imperfections :

1. First rule : Tone - From light to dark.

2. Second rule : Roughness - From rough to smooth, via rippled.

3. Third rule : Light aspect - From matte to speckled.

4. Fourth rule : Finesse - From fine to coarse.

5. Fifth rule : Regularity - From regular to irregular.

The trend of anomalie influence on visual texture is currently more light, more rough, more speckled, more coarse and more irregular.

\section{CONCLUSION AND PERSPECTIVES}

We present a new generic and reduced classification of aesthetic anomalies from syntactic surface texture analysis and human perception. We show a comparison between geometrical dimensional considerations like an analysis of anomaly origin and appearance and perceptual analysis like an analysis of anomaly visual impact.

Perspectives in the short or medium terms are to identify the different scales in visual perception visual texture indicators and integrate the human perception of values described by the laws of Gelstat, through perception indicators, to improve automatic assessment of aesthetic anomalies according to criteria of human perception.

\section{ACKNOWLEDGMENTS}

We thank our SYMME partners in MESURA project as well as Savoie Mont Blanc Industries and Conseil General 74 to enable us to carry out this research by giving us resources.

\section{REFERENCES}

[1] M. Pointer, "A framework for the measurement of visual appearance," CIE Publication 175-2006: CIE TC1-65 Technical Report, 2006.

[2] ISO-8785, "Geometrical product specifications (gps) - surface imperfections — terms, definitions and parameters," 1998.

[3] J.-C. Krynicki, "Introduction to soft metrology," in XVIII IMEKO World Congress, 2006.

[4] C. Eugène, "Measurement of "total visual appearance" a cie challenge of soft metrology" in 12th IMEKO TC1 $\&$ TC7 Joint Symposium on Man, Science $\&$ Measurement, pp. 61-65, 2008.

[5] A. S. Guerra, M. Pillet, and J.-L. Maire, "Control of variability for man measurement," in 12th IMEKO TC1-TC7 joint Symposium on Man, Science and Measurement, p. nc, 2008.

[6] N. Baudet, J.-L. Maire, and M. Pillet, "The visual inspection of product surfaces," Food Quality and Preference 27(2), pp. 153-160, 2013.

[7] S.-F. Désage, G. Pitard, M. Pillet, H. Favrelière, J.-L. Maire, F. Frelin, S. Samper, and G. Le Goïc, "Extended visual appearance texture features," in ISET/SPIE Electronic Imaging 2015, 9394 - 9398, February 2015.

[8] T. S. Nguyen, M. Avila, and S. Begot, "Automatic detection and classification of defect on road pavement using anisotropy measure," in European Signal Processing Conference, pp. 617-621, 2009. 
[9] O. Kleynen, V. Leemans, and M.-F. Destain, "Development of a multi-spectral vision system for the detection of defects on apples," Journal of Food Engineering 69(1), pp. 41-49, 2005.

[10] R. Stojanovic, P. Mitropulos, C. Koulamas, Y. Karayiannis, S. Koubias, and G. Papadopoulos, "Real-time vision-based system for textile fabric inspection," Real-Time Imaging 7(6), pp. 507-518, 2001.

[11] S.-F. Désage, G. Pitard, M. Pillet, H. Favrelière, F. Frelin, S. Samper, G. Le Goïc, L. Gwinner, and P. Jochum, "Visual quality inspection and fine anomalies: Methods and application," in Precision Assembly Technologies and Systems, pp. 94-106, Springer, 2014.

[12] G. L. Goic, H. Favreliere, S. Samper, and F. Formosa, "Multi scale modal decomposition of primary form, waviness and roughness of surfaces," Scanning 33(5), pp. 332-341, 2011.

[13] A. S. Guerra, Métrologie sensorielle dans le cadre du contrôle qualité visuel. PhD thesis, Université de Savoie, 2008.

[14] J. Maire, M. Pillet, and N. Baudet, "Gage r2\&e2: an effective tool to improve the visual control of products," International Journal of Quality \& Reliability Management 30, pp. 161-176, Jan. 2013.

[15] M. Haindl and J. Filip, Visual Texture: Accurate Material Appearance Measurement, Representation and Modeling, Springer Science \& Business Media, Jan. 2013.

[16] A. Porebski, Sélection d'attributs de texture couleur pour la classification d'images. Application à l'identification de défauts sur les décors verriers imprimés par sérigraphie. $\mathrm{PhD}$ thesis, Université Lille 1, 2009 . 